\title{
Magnetic Field Modulation of Chirooptical Effects in Magnetoplasmonic Structures
}

Gaspar Armelles, ${ }^{\mathrm{a}^{*}}$ Blanca Caballeroa, Patricia Prieto ${ }^{\mathrm{a}}$, Fernando García ${ }^{\mathrm{a}}$, Alfonso Cebolladaa , María Ujué González a and Antonio García-Martina

In this work we analyse the magnetic field effects on the chirooptical properties of magnetoplasmonic chiral structures. The structures consist of two-dimensional arrays of Au gammadions in which thin layers of Co have been inserted. Due to the magnetic properties of the $\mathrm{Au} / \mathrm{Co}$ interface the structures have perpendicular magnetic anisotropy which favours magnetic saturation along the surface normal, allowing magnetic field modulation of the chirooptical response with moderate magnetic fields. These structures have two main resonances. The resonance at 850 $\mathrm{nm}$ has a larger chirooptical response than the resonance at $650 \mathrm{~nm}$, which, on the other hand, exhibits a larger magnetic field modulation of its chirooptical response. This dissimilar behaviour is due to the different physical origin of the chirooptical and magneto-optical responses. Whereas the chirooptical effects are due to the geometry of the structures, the magneto-optical response is related to the intensity of the electromagnetic field in the magnetic (Co) layers. We also show that the optical chirality can be modulated by the applied magnetic field, which suggests

\footnotetext{
aMM-Instituto de Microelectrónica de Madrid (CNM-CSIC)

Isaac Newton 8, PTM, E-28760, Tres Cantos, Madrid, Spain

E-mail: gaspar@imm.cnm.csic.es
} 
that magnetoplasmonic chiral structures could be used to develop new strategies for chirooptical sensing.

\section{Introduction}

In the last few years, a new family of chiral materials based on metallic nanostructures has been developed.[1,2,3,4,5] In these systems, high values of chirooptical effects have been obtained and structures with negative refraction behaviour ${ }^{[6]}$ and sensors with enhanced chiral sensitivity have been realized.[7] At the same time, structures with plasmon resonances and magnetooptical (MO) activity have also been studied. ${ }^{[8]}$ In these so called magnetoplasmonic systems high values of MO activity may be achieved upon plasmon excitation. $[9,10,11,12]$ Moreover, due to their magneto-optical activity, plasmonic properties can be modulated under the action of an external magnetic field..13] These two effects find application niches in, for example, sensing or telecommunications. ${ }^{[14,15,16,17,18,13]} \mathrm{A}$ magnetic field applied along the propagation direction of a circularly polarized light traveling in a chiral medium, does not modify the polarization state of the light and thus it induces no coupling between the eigenmodes of the chiral media.[19,20,21,22,23] Therefore, it is conceivable that plasmonic structures presenting simultaneously optical activity and magneto-optical properties are potential candidates to develop tuneable chiral structures whose properties could be controlled by a magnetic field. The aim of the present work is to analyse this concept by fabricating and studying magnetoplasmonic chiral elements based on gammadion crosses. The choice of these structures is based on the fact that gold gammadions have already shown strong chirooptical effects caused by plasmon excitation.[24,25,26,27] Besides, MO activity can be easily incorporated to these 
systems by introducing layers of ferromagnetic metals inside the $\mathrm{Au}$ crosses (magnetoplasmonic gammadions). Additionally, by choosing the adequate ferromagnetic material and internal layering, the magnetic properties and magnetic field dependence of the signal can be controlled, thus reducing the magnetic field needed to obtain the desired magnetic modulation of the chiral response. We will show that these systems present two main resonances in the spectral range of $400 \mathrm{~nm}-1100 \mathrm{~nm}$, with different chirooptical response and magnetic field induced effects. The physical origin of these two properties is related to the geometry of the structures and the intensity of the in-plane electromagnetic field in the Co MO active layers, respectively.

\section{Results and discussion}

The studied structures consist of two-dimensional arrays of gammadions deposited on glass substrates, whose nominal dimensions and period are given in figure 1a. The internal structure of the magnetoplasmonic gammadion crosses is constituted by a Au/Co multilayer (ML) (12x (6 nm Au / $1 \mathrm{~nm} \mathrm{Co})$ ) covered with $20 \mathrm{~nm}$ of Au (total thickness $104 \mathrm{~nm}$ ). Arrays of 104nm thick pure Au gammadions were also fabricated with the same dimensions and period. In both cases a $2 \mathrm{~nm}$ thick Ti layer was deposited on top of the glass substrate to increase the adhesion. In Figure 1b we present a SEM image of a collection of gammadions as well as an AFM zoom of one of them, showing their specific morphology. As for the purely magnetic properties is concerned, interface anisotropy in the $\mathrm{Au} / \mathrm{Co}$ MLs leads to perpendicular magnetic anisotropy which favours magnetic saturation of the system along the surface normal with moderate magnetic fields. Chiral objects show different absorption for left- and right-hand circularly polarized light, 
property denoted as circular dichroism, CD. The sign of CD depends on the twist or handedness of the structure. MO structures also present CD induced by the magnetic field. In the case of ferromagnetic systems, as the ones studied here, the magnetic field induced CD (magnetic circular dichroism, MCD) depends on the magnetization $(M)$, and is defined as: $M C D=C D(+M)-C D(-M)$, i.e. the difference in the CD signal for the system magnetized in opposite directions. In figure $1 \mathrm{c}$, we present the magnetic field dependent component of the CD signal at a wavelength of $650 \mathrm{~nm}$ as a function of the external magnetic field applied perpendicular to the sample plane (z-axis). The obtained CD hysteresis loop is characteristic of a ferromagnetic structure with perpendicular magnetic anisotropy, with relatively low saturation field and $100 \%$ remanence.

In figure 2 we present experimental and theoretical (insets) CD spectra for pure Au (figure $2 \mathrm{a}$ ) and $\mathrm{Au} /$ Co ML (figure $2 \mathrm{~b}$ ) chiral structures with nominally identical dimensions and the same handedness. As it can be observed, the theoretical and experimental spectra of the Au structure look very similar, with two main features, located at around $650 \mathrm{~nm}$ and $850 \mathrm{~nm}$. The origin of these two features has been attributed to pseudo propagating plasmons excited by the light due to the grating periodicity.[26] The low energy structure is related to the coupling at the grating/air interface, whereas the high energy one is related to the coupling at the grating/substrate interface. In figure 1 of the supplementary information we present the spatial distribution of the electromagnetic (EM) field calculated at the gammadion/substrate interface $(650 \mathrm{~nm})$ and on top of the gammadion structure $(850 \mathrm{~nm})$. The strong increase of the EM intensity near the gammadion structure corroborates the plasmonic character of these features. Moreover, in that figure we also present the $\mathrm{z}$ dependence of the field intensity at the green points, 
corresponding to the maximum of the electromagnetic field intensity. As it can be seen for the high energy feature the EM field is more intense in the gammadion/substrate interface than in the gammadion/air one; the contrary occurs for the low energy feature, where the electromagnetic field intensity is higher at the gammadion/air interface. Moreover, the EM field at $650 \mathrm{~nm}$ is more localized in the gammadion than that at $850 \mathrm{~nm}$.

Compared to the CD of the pure Au system, the main effect of introducing Co is to reduce the relative intensity of the $650 \mathrm{~nm}$ feature with respect to the $850 \mathrm{~nm}$ one, which can be attributed to the different localization of the electromagnetic field for these two features. On the other hand, due to the fabrication process, the actual structures do not have straight edges and sharp corners, presenting in fact more rounded shapes (see Figure 1b). To get insight into the implications of this rounding of the actual shape, in the inset of figure $2 \mathrm{~b}$ we also present a theoretical calculation of a $\mathrm{Au} / \mathrm{Co}$ structure with smoothed edges, the main effect of this smoothing being the reduction of the intensity of the CD signal, with the resonance at $650 \mathrm{~nm}$ more affected by such smoothing, again due to stronger localization of the electromagnetic field for this energy which makes it more sensitive to the changes of the local shape of the structure. Further differences between the experimental and theoretical spectra can be attributed to differences between the experimental and theoretical shapes of the gammadions apart from simple rounding effects (for example fabrication induced local defects or surface roughness).

Once the optical chirality of the fabricated structures has been characterized, the next step is to study its possible modulation by the action of an external magnetic field. In figure 3 we present experimental and theoretical (insets) CD spectra of a 
right-handed (figure 3a) and left-handed (figure 3b) Au/Co ML chiral structures magnetized along the positive (red curves) and negative (black curves) direction of the z-axis, respectively. As expected, a change of sign in the twist direction of the crosses leads to a change of sign in the CD spectra. As intended, due to the presence of a MO active element in the system, the CD spectra also depend on the magnetization direction, and as it can be seen there are spectral regions where the dichroic signals for opposite magnetizations (black and red curves) do not coincide. To highlight the purely magnetic induced effect, we have also plotted in figure 3 the difference between the dichroism spectra for opposite magnetizations (green curves), which corresponds to the magnetic circular dichroism (MCD) signal. This signal has been multiplied by 10. As can be seen, the MCD sign does not depend on the handedness of the structure (the observed dissimilarities between the two samples are related to small morphological variations introduced during the fabrication process). The obtained MCD spectra have features in the same spectral positions than those of the CD spectra, but contrary to the CD signal, the MCD intensity of the high energy structure is larger than the low energy one. This different behaviour is due to the different origin of the CD and MCD signal. Whereas the CD signal is related to the geometry of the structure, the MCD signal is related to the electromagnetic field intensity inside the Co layers. In particular, for very thin Co layers, the MO response is proportional to:[8]

$$
\epsilon_{M O} \int_{V c o} E_{p} E_{S}
$$

where, the integral is calculated in the volume occupied by the Co layers , $\varepsilon_{\text {Mo }}$ is the non diagonal component of the dielectric tensor of Co, $E_{p}\left(E_{s}\right)$ is the p component (s component) of the EM field at the Co layers when the incident light is 
p polarized (s polarized) calculated for a pure $\mathrm{Au}$ structure. Due to the stronger localization of the field inside the nanostructures for the high energy feature, the $E_{p} \times E_{s}$ product is larger in this spectral region than in the low energy one (see supplementary information), which explains the larger MCD around the $650 \mathrm{~nm}$ resonance.

The previous results show that the application of a magnetic field allows the modulation of the chiral activity in magnetoplasmonic chiral structures. Compared to other kinds of magneto-chiral systems considered in the literature, here the presence of a ferromagnetic component enhances the MCD effect, and as a consequence the ratio between the intensity of the MCD and CD features is in the present work between one to two orders of magnitude higher than the same ratio in para- and diamagnetic magneto-chiral systems for equivalent magnetic fields.[28,29,30]

This effect may allow developing new sensing strategies by making use of the magnetic field modulation of the chirality (C) of the electromagnetic (EM) field (socalled optical chirality) in this kind of systems. The local optical chirality, C(r), is given by $C(\boldsymbol{r})=-\frac{\varepsilon_{0} \omega}{2 C_{0}} \operatorname{Im}\left(\boldsymbol{E}^{*}(\boldsymbol{r}) \cdot \boldsymbol{B}(\boldsymbol{r})\right),[31,32,33,34,35]$ where $\omega$ is the frequency of the EM field, $\mathbf{E}(\mathbf{r})$ and $\mathbf{B}(\mathbf{r})$ are the electric and magnetic components of the EM field at $\mathbf{r}$, and $\mathrm{C}_{0}$ is a normalization constant corresponding to the modulus of the optical chirality for a left (right) circular polarized wave propagating in vacuum $\left(C_{0}=\left|C^{L C P}\right|=\left|C^{R C P}\right|\right)$. For example, the chirooptical response of a molecule depends on the difference of this optical chirality $\mathrm{C}$ for left- and right-handed circularly polarized light, a difference that can be enhanced if the molecule is adsorbed on a chiral-plasmonic nanostructure. ${ }^{[7]}$ The local optical chirality depends strongly on the geometrical shape and arrangement of the nanostructures 
and enhancement factors higher than 100 have been calculated in the near field region of chiral oligomer structures.[34] In the left column of figure 4 we present the local optical chirality calculated (with no magnetic effects included) at a wavelength of $850 \mathrm{~nm}$ (and at $5 \mathrm{~nm}$ above the surface) for right-handed magnetoplasmonic gammadions illuminated by right-handed circularly polarized light (figure 4 a) and left-handed circularly polarized light (figure 4 b). Figure $4 \mathrm{c}$ shows the difference of those optical chirality plots, obtaining local enhancements factors of about 5 . In our case, the local chirality is also affected by the orientation of the magnetization of the Co layers, thus we present in figures $4 \mathrm{~d}$, e, $\mathrm{f}$ the difference of the optical chiralities for Co layers magnetized in opposite directions along the z-axis. As it can be observed in figures $4 \mathrm{~d}$ and e, the magnetic modulation of the optical chirality is larger for left handed circularly polarized light, as also happens for the non-magnetic component of the optical chirality (figures $4 \mathrm{a}$ and $\mathrm{b}$ ). Also, the spatial distribution of both non-magnetic component and magnetic components of the optical chirality are very similar. Additionally, the switching of the Co magnetization from positive to negative can give rise to a relative modulation of the local optical chirality $\left(2 \times\left(C^{\uparrow}-C^{\Downarrow}\right) /\left(C^{\uparrow}+C^{\Downarrow}\right)\right)$ of around $0.2 \%$. Finally, in figure $4 \mathrm{f}$ we show the magnetic field modulation of the difference of optical chiralities, presenting relative modulation values similar to those obtained above. Concerning the potential use for sensing of the $650 \mathrm{~nm}$ feature, the corresponding results also at $5 \mathrm{~nm}$ above the surface are also shown in the supplementary information. As it can be seen in this case, all the values are lower than for $850 \mathrm{~nm}$ because the electromagnetic field is concentrated now near the substrate/gammadion interface.

As we have shown, by inserting Co layers in Au gammadions it is possible to obtain 
novel systems where both chiral and MO properties coexist. The structural helicity of these elements gives rise to a sizeable chirooptical activity (CD), in addition to which a MO activity (MCD), due to the presence of Co, is also present. Because of the periodicity of the fabricated structures, the incoming light couples to two pseudopropagating plasmons localized at the substrate/gammadion and gammadion/air interfaces, which manifest as two spectral features located at high and low energy respectively. Interestingly, the different degree of localization of the electromagnetic field for these two modes strongly determines their chirooptical and magnetooptical activities, as well as their sensitivity to the insertion of Co or to morphological modifications. Actually, the high energy feature is characterized by a stronger concentration of the EM field in the gammadions, and as a consequence it is more sensitive to the insertion of $\mathrm{Co}$, the application of a magnetic field and the presence of defects due to fabrication effects. We have also shown that, due to the presence Co, the local optical chirality depends on the magnetic field, which suggest that both $C D$ and MCD signals could be sensitive to the chiral component of molecules adsorbed on top of the nanostructure. Measuring both magnitudes in only one chiral nanostructure may allow to discriminate the changes induced by the chiral component of the molecule from the changes induced by the non chiral part of the molecule. This could give rise to novel strategies for chirooptical sensing using chiral magnetoplasmonic structures.

\section{Conclusions}

In conclusion, we have demonstrated magnetic field induced changes of chirooptical effects in magnetoplasmonic gammadions. This is achieved by the incorporation of thin ferromagnetic Co layers into a pure $\mathrm{Au}$ chiral structure. 
These systems present two main resonances in the spectral range of $400 \mathrm{~nm}$ $1100 \mathrm{~nm}$, with different chirooptical response and magnetic field induced effects whose physical origin is related to the geometry of the structures and the intensity of the in-plane electromagnetic field in the Co MO active layers, respectively. For equivalent magnetic fields, the relative modulation of the chirooptical effects is between one to two orders of magnitude higher than that obtained in para- and dia- magneto-chiral systems. Besides, we show that the optical chirality can also be modulated by the applied magnetic field. This proof of concept opens the route to new chirooptical sensing strategies by designing chiral magnetoplasmonic structures with increased local optical chirality and magnetic field modifications of chirooptical effects.

\section{Acknowledgements:}

Funding from Spanish Ministry of Economy and Competitiveness through grants "FUNCOAT" CONSOLIDER CSD2008-00023, and MAPS MAT2011-29194-C02-01, and from Comunidad de Madrid through grants "NANOBIOMAGNET" S2009/MAT1726 and "MICROSERES-CM" S2009/TIC-1476 is acknowledged. 


\section{Figures:}

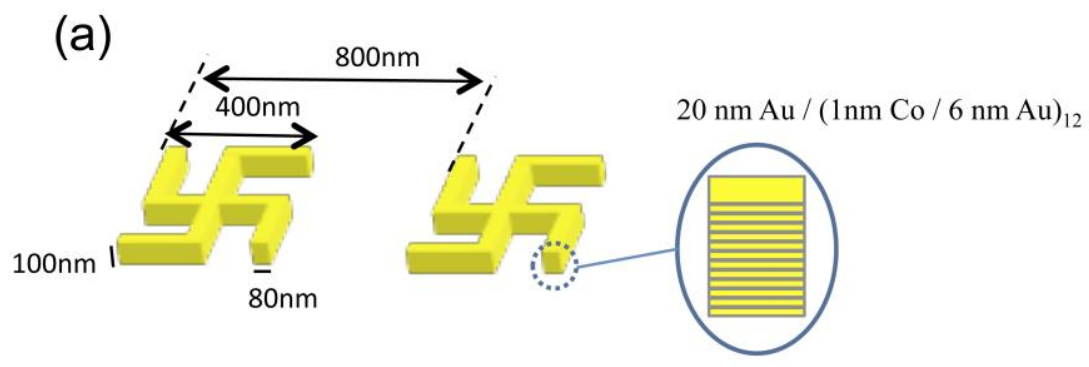

(b)

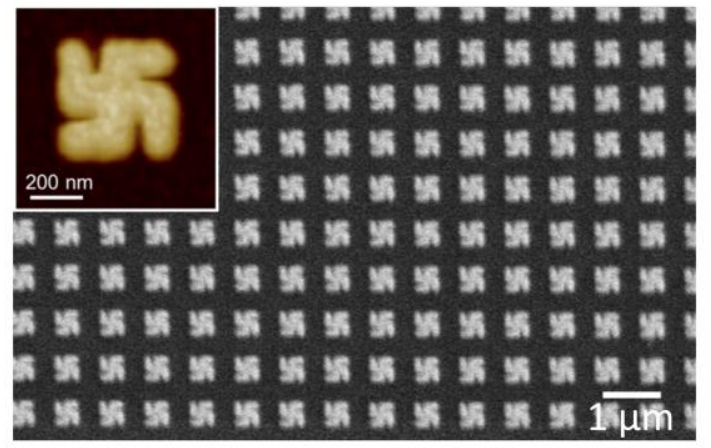

(c)

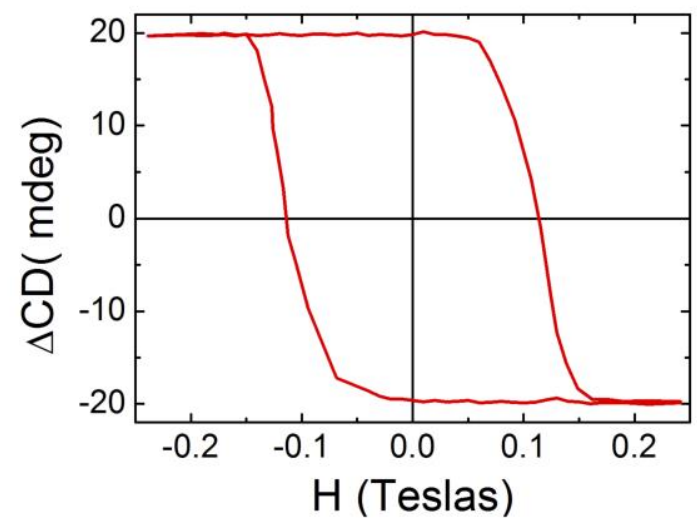

Figure 1. (a): (Left) Schema with the dimensions and period of the $\mathrm{Au}$ and $\mathrm{Au} / \mathrm{Co}$ multi-layered gammadion crosses, (right) cross-section of the internal structure of the magnetoplasmonic Au/Co gammadions. (b): SEM image of a representative array. The inset shows an AFM zoom of one of the gammadions. (c): Magnetic field dependence of the circular dichroism signal for the $\mathrm{Au} / \mathrm{Co}$ multilayer crosses. 


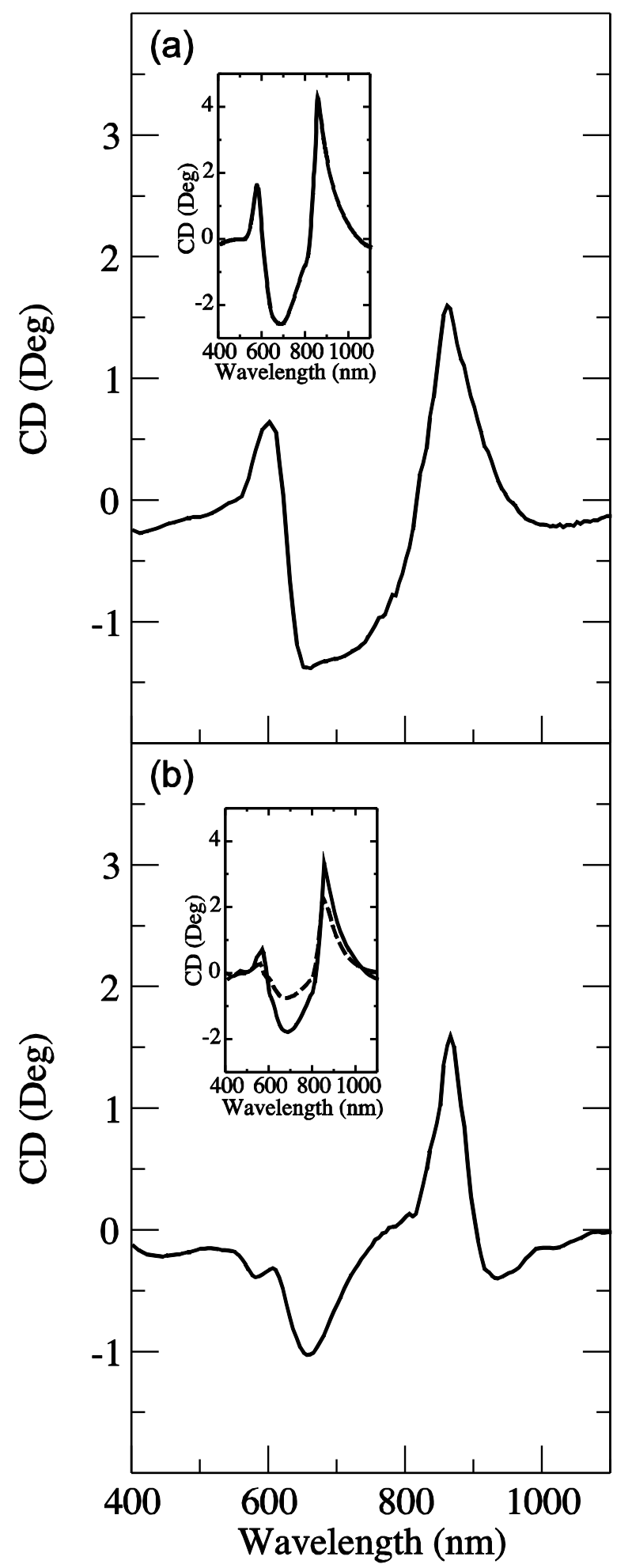

Figure 2. Experimental circular dichroism spectra of $\mathrm{Au}(\mathrm{a})$ and $\mathrm{Au} / \mathrm{Co}(\mathrm{b})$ gammadions arrays. The insets show theoretical spectra for perfect gammadion crosses (full line) and gammadion crosses with smooth edges (dotted line). 


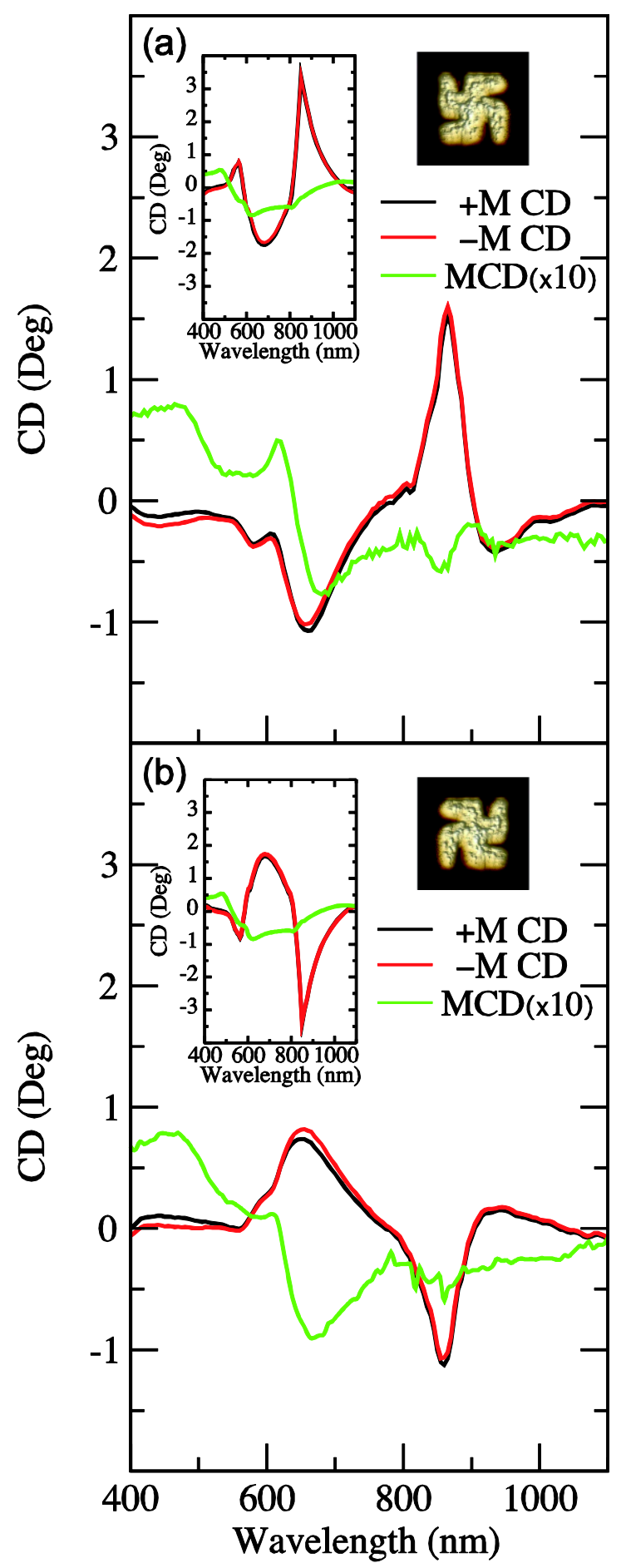

Figure 3. Experimental and theoretical (insets) circular dichroism spectra of a right-handed (a) and left handed (b) Au/Co chiral structures, magnetized along the positive (black curves) and negative (red curves) direction of the z-axis, respectively. The green curves correspond to the magnetic circular dichroism (MCD) spectra. The MCD signal has been multiplied by 10. 
$850 \mathrm{~nm}$

Non-magnetic component Magnetic component
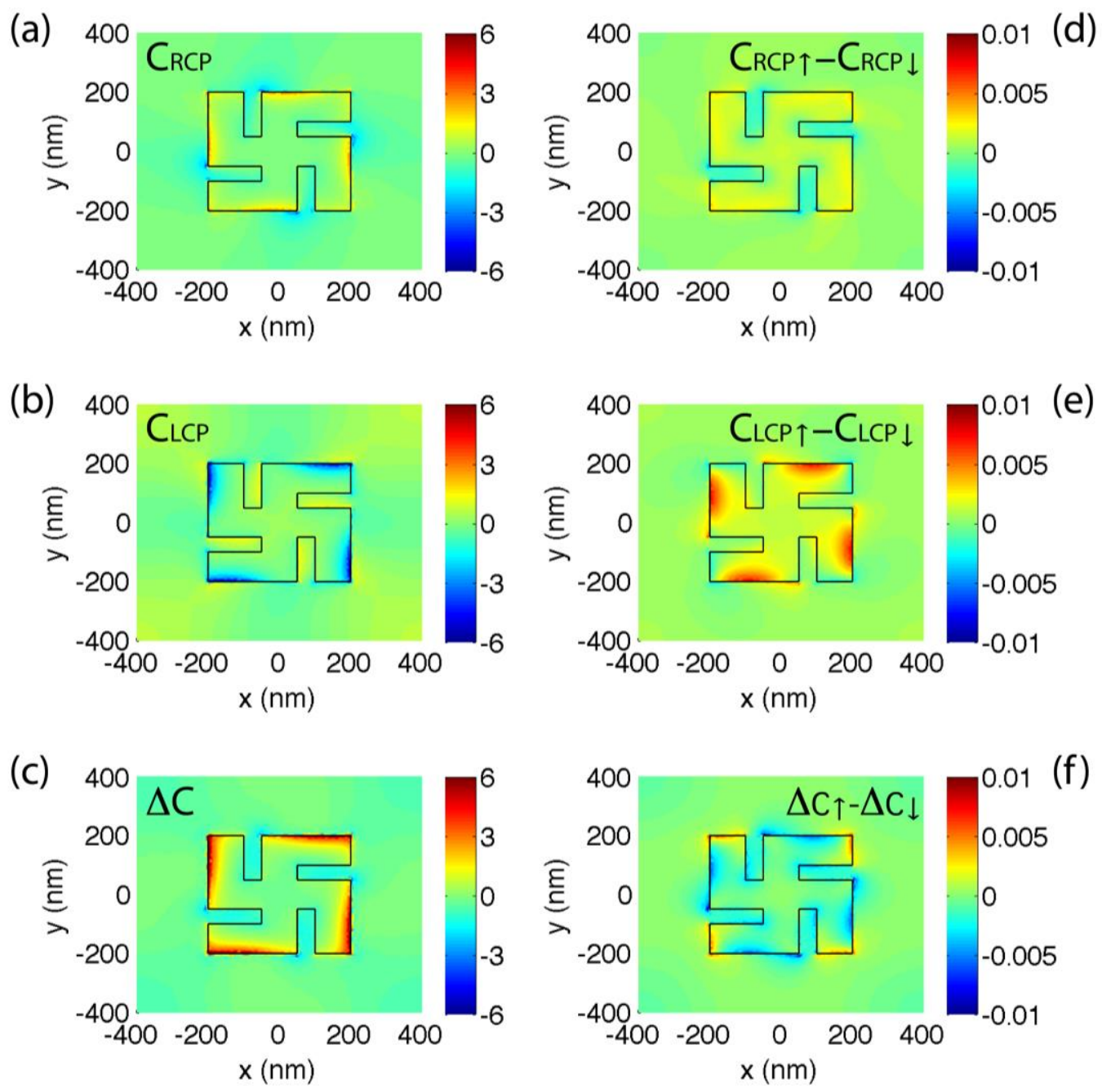

Figure 4. (Left column) Calculated optical chirality plots calculated at $850 \mathrm{~nm}$ and at $5 \mathrm{~nm}$ above the gammadions surface when they are illuminated with: (a) righthanded circularly polarized light, (b) left right-handed circularly polarized light, (c) difference between the optical chirality in plots a and b. (Right column)

Difference between the optical chirality when the structures are magnetized along the positive and negative direction of the z-axis, respectively, for illumination with (d) right-handed circularly polarized light, (e) left right-handed circularly polarized light. (f) Depicts the magnetic component of the optical chirality depicted in (c). 


\section{Notes and references:}

[1] Z. Fan and A.O. Govorov, Nano Lett., 2010, 10, 2580.

[2] A.Guerrero-Martinez, J.L. Alonso-Gomez, B. Auguie, M.M. Cid and L.M. LizMarzan, NanoToday, 2011, 6, 381.

[3] A. Christofi, N. Stefanou, G. Gantzounis and N. Papanikolaou, J. Phys. Chem C, 2012, 116, 16674.

[4] M.Hentschel,M. Schäferling, T. Weiss,N. Liu and H. Giessen, Nano Lett., 2012, 12, 2542.

[5] V.K. Valev, J.J. Baumberg, C. Sibilia and T. Verbiest, Adv. Mat., 2013, 25,2517.

[6] Z. Li, M. Mutlu and E. Ozbay, J. Opt, 2013, 15, 023001.

[7] E. Hendry, T. Carpy, J. Johnston, M. Popland, R.V. Mikhaylovskiy, A.J. Lapthorn, S.M. Kelly, L.D. Barron, N. Gadegaard and M. Kadodwala, Nat. Nanotech., 2010, 5, 783.

[8] G. Armelles, A. Cebollada, A. García-Martín and M. U. González, Adv. Opt. Mater., 2013, 1, 10.

[9] C. Hermann, V. A. Kosobukin, G. Lampel, J. Peretti, V. I. Safarov and P. Bertrand, Phys. Rev. B,2001, 64, 235422.

[10] J. B. González-Díaz, A. García-Martín, J. M. García-Martín, A. Cebollada, G. Armelles, B. Sepúlveda, Y. Alaverdyan and M. Käll, Small, 2008, 4, 202.

[11] V. I. Belotelov, I. A. Akimov, M. Pohl, V. A. Kotov, S. Kasture, A. S. Vengurlekar, A. V. Gopal, D. R. Yakovlev, A. K. Zvezdin and M. Bayer, Nat. Nanotechnol., 2011, 6, 370.

[12] J. Chin, T. Steinle, T Wehlus, D Dregely, T. Weiss, V.L. Belotelov, B. Stritzker and H. Giessen, Nat. Comm., 2013, 4, 1599.

[13] V. V. Temnov, G. Armelles, U. Woggon, D. Guzatov, A. Cebollada, A. GarciaMartin, J. M. Garcia-Martin, T. Thomay, A. Leitenstorfer and R. Bratschitsch, Nat. Photonics, 2010, 4, 107.

[14] B. Sepúlveda, A. Calle, L. M. Lechuga and G. Armelles , Opt. Lett., 2006 , 31,1085.

[15] D. Regatos, D. Fariña, A. Calle, A. Cebollada, B. Sepúlveda, G. Armelles and L. M. Lechuga, J. Appl. Phys., 2010, 108, 054502.

[16] M. G. Manera, G. Montagna, E. Ferreiro-Vila, L. González-García, J. R. SánchezValencia, A. R. González-Elipe , A. Cebollada, J. M. García-Martín, G. Armelles and R. Rella, J. Mater. Chem., 2011, 21, 16049.

[17] V. Bonanni, S. Bonetti, T. Pakizeh, Z. Pirzadeh, J. Chen, J. Nogues, P. Vavassori, R. Hillenbrand, J. Akerman and A. Dmitriev, Nano Lett. 2011, 11, 5333

[18] D. Martín-Becerra, G. Armelles, M.U. Gónzalez and A.M García-Martin, New J. Phys., 2013, 15, 085021.

[19] N. Engheta, D.L. Jaggard and M.K. Kowarrz, IEEE Trans. on Antenas and

Propagation, 1992, 40, 367.

[20] H. Tauk, J. Opt. Soc. Am. A, 1997, 14, 2006.

[21] A.H. Gevorgyan, Mol. Cryst. Liq. Cryst, 2002, 382, 1.

[22] A.H. Gevorgyan. Tech. Phys., 2002, 471008.

[23] I. Bita and E.L. Thomas, J. Opt Soc. Am B, 2005, 22,1199.

[24] T. Vallius, K. Jefimovs, J. Turunen, P. Vahimaa and Y. Svirko, Appl. Phys. Lett, 2003, 83,234.

[25] M.Kuwata-Gonokami, N. Saito, Y. Ino, M. Kauranen, K. Jefimovs, T. Vallius, J. Turunen and Y. Svirko, Phy. Rev. Lett, 2005, 95, 227401. 
[26] K. Konishi,T. Sugimoto, B.Bai, Y. Svirko and K. Kuwata-Gonokami, Opt Express, 2007, 15, 9575.

[27] B. Bai, Y. Svirko,J. Turunen, and T. Vallius, Phys. Rev. A, 2007, 76, 023811.

[28] G.L. Rikken and E. Raupach. Phys. Rev E, 1998, 58, 5081.

[29] Y. Kitagawa, H. Segawa and K. Ishii, Angew.Chem. Int. Ed., 2011, 50, 9133.

[30] Y. Kitagawa, H. Segawa and K. Ishii, Chem, Commun., 2012, 48, 5091.

[31] Y. Tang and A.E. Cohen, Science, 2011, 332, 333.

[32] Y. Tang and A.E. Cohen, Phys. Rev. Lett., 2010, 104, 163901.

[33] E.Hendry, R.V. Mikhaylovskiy, L.D. Barron, M. Kadodwala and T.J. Davis, Nano Lett., 2012, 12, 3640.

[34] M. Schaferling, D. Dregely,M. Hentschel and H. Giessen, Phys. Rev X, 2012, 2, 031010.

[35] M. Schaferling, X. Yin and H. Giessen, Opt. Express, 2012, 20, 26326. 


\section{Supplementary Information}

Samples fabrication. The gammadions arrays were fabricated by e-beam lithography and etching. First, continuous films were grown with combined electron beam (Co, Ti) and thermal (Au) evaporation. Then, a $100 \mathrm{~nm}$ thick layer of electron sensitive negative resist (AR-N7500.08) was spin-coated on top of the metallic films and the chiral crosses were patterned in areas of $400 \mathrm{x} 400 \mu \mathrm{m}^{2}$. After development, the pattern was transferred to the metal layers by Ar milling and the remaining resist was removed by using $\mathrm{O}_{2}$ reactive ion etching.

Optical characterization. Circular dichroism (CD), both pure optical and magnetic CD (MCD) has been measured in the spectral range $450 \mathrm{~nm}-1100 \mathrm{~nm}$ using a photoelastic modulator and locking amplifier techniques. The polarization state of a linear polarized monochromatic light was modulated at $42 \mathrm{kHz}$ by a photoelastic modulator and, after traversing the sample, analysed by a linear polarizer mounted on a rotation stage and detected by a photomultiplier or a GaInAs diode. The transmission spectra were measured at normal incidence. With this experimental set-up we obtained the real and imaginary part of $t_{s p} / t_{s s}\left(\right.$ or $\left.t_{p s} / t_{p p}\right)$, where $t_{s s}\left(t_{p p}\right)$ are the transmission coefficients of the structure for $s(p)$-polarized light and $t_{s p}$ $\left(t_{p s}\right)$ the polarization conversion factors. At normal incidence, and for small values of $t_{p s}$, the imaginary part of $t_{s p} / t_{p p}$, denoted ellipticity, $\varepsilon$, is related to CD as: $2 \varepsilon=\frac{I^{R C P}-I^{L C P}}{I^{R C P}+I^{L C P}}$. For determining MCD, the magnetic field was applied perpendicular to the sample plane using permanent magnets. The magnets were mounted on translational and rotational stages, which allowed controlling the intensity and direction of the applied magnetic field. Following the procedure described above, CD for the magnetization applied in one sense and for reversed magnetization can 
be obtained, and from that the magnetic circular dichroism is calculated, MCD = $\mathrm{CD}(+\mathrm{M})-\mathrm{CD}(-\mathrm{M})$

Theoretical calculations. The theoretical CD spectra and field distributions were computed using the finite elements commercial software COMSOL Multiphysics. Regarding the materials properties, the permittivities of the metals used in the calculations were obtained from ellipsometric measurements of $\mathrm{Au}$ and $\mathrm{Co}$ continuous films, while the glass substrate refractive index was set to be 1.5 . For the $\mathrm{Au} / \mathrm{Co}$ gammadion we average the multilayer out so that the $12 \mathrm{Au} / \mathrm{Co}$ bilayers were replaced in the simulation by one gammadion-shaped layered of $84 \mathrm{~nm}$ height with an average effective dielectric tensor $\varepsilon_{\mathrm{eff}}=\left(6^{*} \varepsilon_{\mathrm{Au}}+1^{*} \varepsilon_{\mathrm{Co}}\right) / 7$. 
(a)

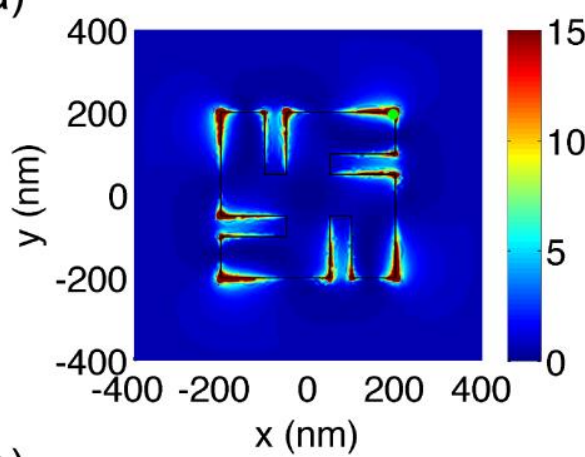

(b)

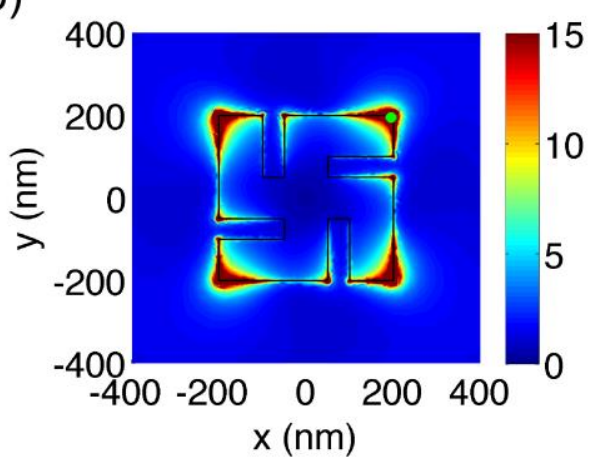

(c)

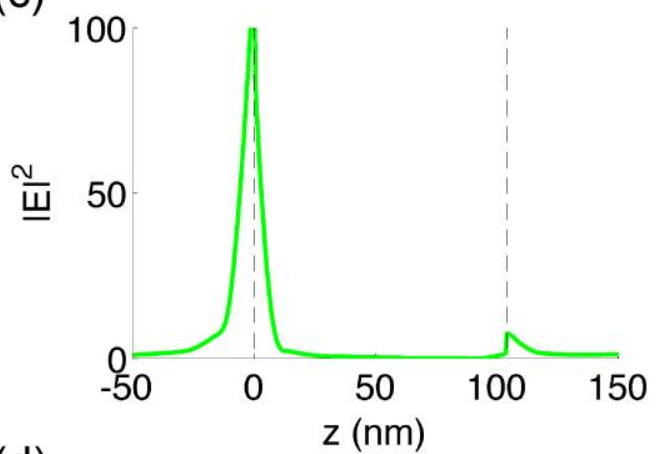

(d)

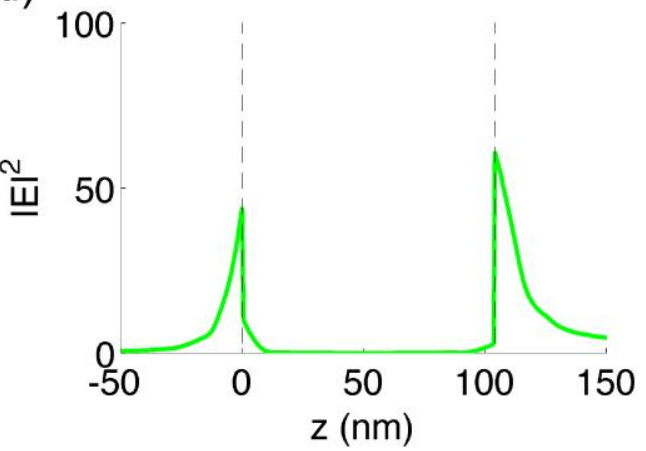

Figure SI-1: (a) In-plane electromagnetic field distribution calculated at $5 \mathrm{~nm}$ below the substrate/gammadion interface for a wavelength of $650 \mathrm{~nm}$ the substrate (b) In-plane electromagnetic field distribution calculated at $5 \mathrm{~nm}$ above the gammadion/air interface for a wavelength of $850 \mathrm{~nm}$ the substrate. z-dependence calculated at the green point for $650 \mathrm{~nm}$ (c) and $850 \mathrm{~nm}(\mathrm{~d})$ ( $\mathrm{z}=0$ corresponds to the substrate/gammadion interface). 
The spectral dependence of the modulus of $\mathrm{E}_{\mathrm{p}}{ }^{*} \mathrm{E}_{\mathrm{s}}$ integrated to all the Co layers is much larger in the region corresponding to the high energy feature than in that related to the low energy one. This explains the larger MCD in the high energy region. The value of MCD is proportional to this product, convoluted by the spectral dependence of the modulus of $\varepsilon_{\mathrm{MO}}$, which modifies slightly the overall spectral dependence of MCD.

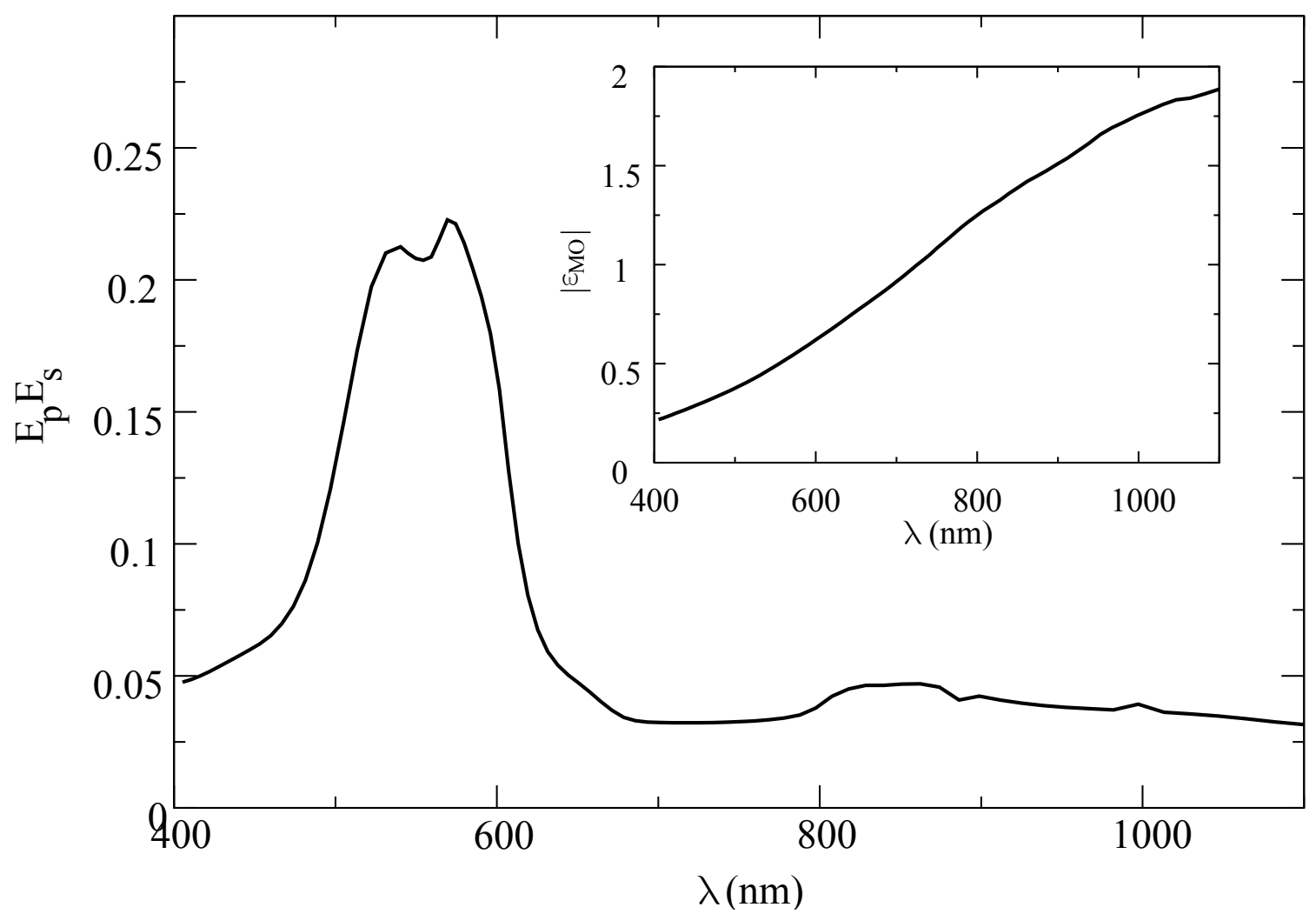

Figure SI-2: Spectral dependence of the modulus of $E_{p} E_{s}$ integrated over all the Co layers. The inset shows the spectral dependence of the modulus of the magnetooptical constant ( $\left.\varepsilon_{\mathrm{MO}}\right)$ of Co. 
$650 \mathrm{~nm}$
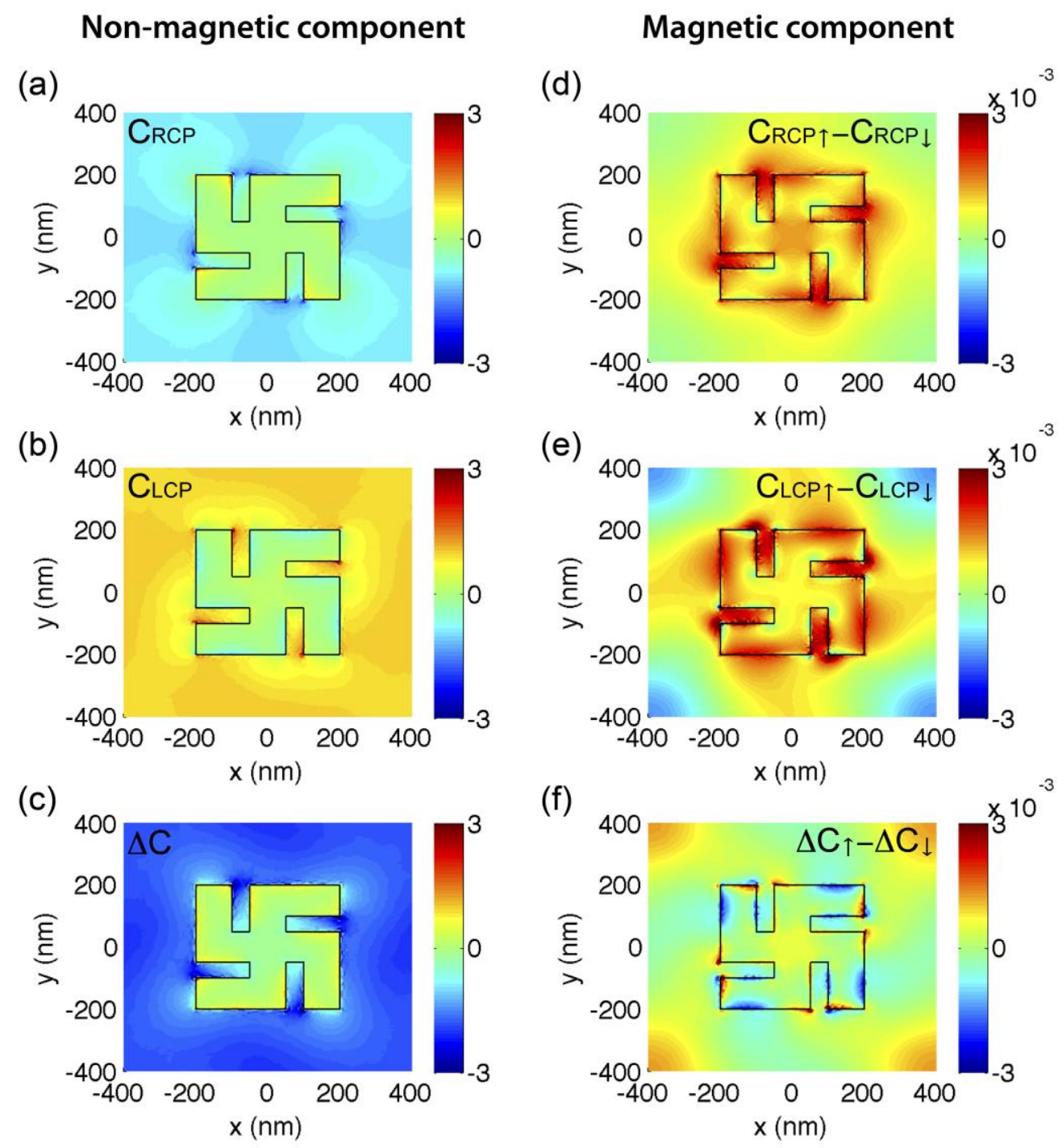

Figure SI-3 (Left column) Calculated optical chirality plots calculated at 650nm and at $5 \mathrm{~nm}$ above the gammadions surface when they are illuminated with: (a) righthanded circularly polarized light, (b) left right-handed circularly polarized light, (c) difference between the optical chirality in plots a and b. (Right column) Difference between the optical chirality when the structures are magnetized along the positive and negative direction of the z-axis, respectively, for illumination with (d) right-handed circularly polarized light, (e) left right-handed circularly polarized light. (f) Depicts the magnetic component of the optical chirality depicted in (c). 